\section{Management of ophthalmologic manifestations of mitochondrial diseases}

To the Editor: We would like to thank the Mitochondrial Medicine Society and the authors of the recently published consensus article in Genetics in Medicine regarding patient care standards for primary mitochondrial-disease patients ${ }^{1}$ for their important contribution to the literature, which will be used by experts and general practitioners alike in the care of mitochondrial patients. Although we completely understand the challenge of covering in detail and offering expert advice on the multitude of systemic and neurological manifestations of mitochondrial diseases in one set of guidelines, the ophthalmologic manifestations of mitochondrial disorders are particularly difficult to address in a forum such as this. We agree with the authors that the particular ophthalmologic findings in patients with generalized mitochondrial disorders are distinctive and require different diagnostic and management approaches from those related to the primary hereditary optic neuropathies of mitochondrial origin, such as Leber hereditary optic neuropathy (LHON). As neuro-ophthalmologists with a particular expertise and interest in these disorders, we offer the following comments, suggestions, and questions.

First, regarding the second of the Ophthalmology Recommendations ("Patients should be referred to a neuroophthalmologist for detection of visual, retinal, macular, and optic nerve changes at the time of diagnosis, supported by optical coherence tomography and electroretinography. If symptomatic, an assessment of intraocular pressure is indicated. Annual ophthalmology exams should be conducted thereafter"), general mitochondrial patients need not be sent specifically to a neuro-ophthalmologist, although neuroophthalmologists are often indeed the best gatekeepers for the vision-related aspects of these disorders. Initially, either a general ophthalmologist or a neuro-ophthalmologist should be sufficient to provide appropriate triage to the correct ophthalmologic specialist if there is a clinical need, such as a retina specialist for patients with maculopathy or pigmentary retinopathy, an oculoplastics specialist for patients requiring possible ptosis surgery, a cornea specialist for patients with exposure keratopathy, or a strabismus specialist for patients with diplopia (although the majority of patients with diplopia can be managed satisfactorily with prisms and strabismus surgery is rarely indicated, except perhaps for short-term cosmetic alignment). Optical coherence tomography is only one of many ancillary tests available to the ophthalmologist, and it is not particularly indicated in the diagnosis of these patients, although it may be helpful in following them over time. We agree that an electroretinogram may be helpful in diagnosis when a retinopathy is suspected, but the retinal pigmentary disturbance is usually clinically apparent on dilated ocular funduscopy in the majority of patients. As an alternative to visual electrophysiology in borderline cases, autofluorescence is a sensitive and more readily available clinical tool to delineate abnormal areas of outer retinal dysfunction. One particular statement in this recommendation, however, does need correction. The assertion "if symptomatic, an assessment of intraocular pressure is indicated," is incorrect. Assessment of intraocular pressure is an integral part of every general eye examination by every ophthalmologist, and elevated intraocular pressure is not found more frequently among mitochondrial-disease patients, nor is it symptomatic unless in the rare settings of acute angle-closure glaucoma or ocular inflammation.

Regarding the fifth ophthalmology recommendation, lubrication of the eyes is necessary not because of "inappropriate eyelid spread of tears in patients with ptosis or after ptosis repair" but rather because of corneal exposure and the concomitant keratopathy that results from a combination of poor eyelid closure after ptosis surgery, weak orbicularis oculi muscle function from myopathic facial weakness causing weak eye closure, and lack of a normal Bell's phenomenon due to chronic progressive external ophthalmoplegia. Furthermore, it is important to ensure that any ptosis surgery is carried out with these considerations in mind rather than purely for cosmesis.

Regarding the sixth ophthalmology recommendation ("Heavy alcohol use and smoking should be avoided among LHON carriers as these are strongly associated with increased risk of visual loss"), although the authors are correct that all LHON carriers should avoid smoking and heavy alcohol use because of the risk of precipitating vision loss, the same recommendation should be made for affected LHON patients, as these exposures may also contribute to worse visual outcomes by further compromising mitochondrial function in the remaining surviving retinal ganglion cells.

Finally, we do not agree with the seventh ophthalmology recommendation ("LHON patients should receive periodic neurologic evaluations and annual ECG"). Periodic neurologic evaluations are not necessary in LHON patients, because the risk of these manifestations is extremely small and the few patients with these complaints will probably bring them to the attention of their care providers. Similarly, most of the cardiac abnormalities presumed to be related to LHON-including cardiac conduction defects, particularly the pre-excitation syndromes, and various forms of usually asymptomatic cardiac hypertrophy-were reported in case series, often involving multiple members of the same family, and not validated across populations. In our experience, which includes long-term 
follow-up of large numbers of patients in the RHODOS study and in the large Brazilian LHON cohort, there does not seem to be an increased risk of life-threatening cardiac arrhythmias among LHON carriers. We have always been under the impression that a single ECG with a rhythm strip was sufficient and that there is no need for yearly ECGs in LHON patients without cardiac-related symptomatology. If the consensus statement's authors with cardiac expertise feel differently, we would be very grateful for the information on which they base their assessment and the underlying rationale.

We would also like to alert the authors, the Mitochondrial Medicine Society members, and the readership of Genetics in Medicine to two additional resources for practitioners, one regarding the optimal management of ophthalmologic issues in mitochondrial-disease patients provided by the group from Newcastle $^{2}$ and the other an international consensus statement on the clinical and therapeutic management of LHON. ${ }^{3}$ We hope this additional information will prove useful.

Thank you again for producing this excellent, informative, and extremely important contribution for mitochondrialdisease physicians and their patients.

\section{DISCLOSURE}

P.Y.-W.-M. is a consultant for GenSight Biologics. N.J.N. is a consultant for GenSight Biologics and was a consultant for Santhera Pharmaceuticals.

\section{ACKNOWLEDGMENTS}

N.J.N. received research support from GenSight Biologics and financial compensation from the Data and Safety Monitoring Board for Quark Pharmaceuticals' Ischemic Optic Neuropathy Trial; departmental support from Research to Prevent Blindness (New York, NY); and funding through NIH grant P30-EY006360 (Core Grant, Department of Ophthalmology). P.Y.-W.-M. is supported by a Clinician Scientist Fellowship Award (G1002570) from the UK Medical Research Council. He also receives funding from Fight for Sight (UK), the UK National Institute of Health Research (NIHR) as part of the Rare Diseases Translational Research Collaboration, and the NIHR Biomedical Research Centre based at Moorfields Eye Hospital NHS Foundation Trust and the UCL Institute of Ophthalmology (the views expressed are those of the author and not necessarily those of the NHS, the NIHR, or the Department of Health). A.A.S. received research support from GenSight Biologics, Stealth BioTherapeutics, and Bio.Electron. R.K. has received research funding from the International Foundation for Optic Nerve Disease, LHON.org, and the United Mitochondrial Disease Foundation. V.C. received research funding from the Italian Ministries of Health and of Research, Telethon-Italy (grant GGP06233), e-RARE, "Programma di Ricerca Regione-Università 2010-2012" (PRUa1RI-2012-008), and patient-led organizations (IFOND, UMDF, MITOCON, the Poincenot Family, and the Gino Galletti Foundation).

Nancy J. Newman, $M D^{1}$, Patrick Yu-Wai-Man, $M D, P h D^{2,3,4}$, Alfredo A. Sadun, $M D, P h D^{5,6}$,

Rustum Karanjia, $M D, P h D^{5,6,7,8}$ and

Valerio Carelli, $M D$, $P h D^{9,10}$

${ }^{1}$ Departments of Ophthalmology, Neurology, and Neurosurgery, Emory University School of Medicine, Atlanta, Georgia, USA; ${ }^{2}$ Cambridge Centre for Brain Repair,

Department of Clinical Neurosciences, and MRC Mitochondrial Biology Unit,

University of Cambridge, Cambridge, UK; ${ }^{3}$ Wellcome Trust Centre for Mitochondrial Research, Institute of Genetic Medicine, Newcastle University, Newcastle upon Tyne, UK; ${ }^{4}$ NIHR Biomedical Research Centre, Moorfields Eye Hospital, and UCL Institute of Ophthalmology, University College London, London, UK; ${ }^{5}$ Doheny Eye Centers, Department of Ophthalmology, David Geffen School of Medicine at UCLA, Los Angeles, California, USA; ${ }^{6}$ Doheny Eye Institute, Los Angeles, California, USA; ${ }^{7}$ Ottawa Eye Institute, University of Ottawa, Ottawa, Ontario, Canada; ${ }^{8}$ Ottawa Hospital

Research Institute, Ottawa, Ontario, Canada; ${ }^{9}$ IRCCS Institute of Neurological Sciences of Bologna, Bellaria Hospital, Bologna, Italy; ${ }^{10}$ Department of Biomedical and Neuromotor Sciences (DIBINEM), University of Bologna, Bologna, Italy.

Correspondence: Nancy J. Newman (ophtnjn@emory.edu)

\section{REFERENCES}

1. Parikh S, Goldstein A, Karaa A, et al. Patient care standards for primary mitochondrial disease: a consensus statement from the Mitochondrial Medicine Society [online only]. Genet Med 2017. doi:10.1038/ gim.2017.107.

2. Newcastle Mitochondrial Disease Guideline Development Group. Ocular Involvement in Adult Mitochondrial Disease: Screening and Initial Management. 2011. http://www.newcastle-mitochondria.com/wpcontent/uploads/2016/03/Ophthalmology-Guidelines.pdf. Accessed 29 July 2017

3. Carelli V, Carbonelli M, de Coo I, et al. International consensus statement on the clinical and therapeutic management of Leber's hereditary optic neuropathy. J Neuroophthalmol (in press).

Advance online publication 26 October 2017. doi:10.1038/gim.2017.171 